\title{
The power of a collectivity to act in weighted voting games with many small voters
}

\author{
Ines Lindner
}

Received: 27 July 2005 / Accepted: 20 May 2007 / Published online: 13 July 2007

(C) Springer-Verlag 2007

\begin{abstract}
We analyze the propensity to approve a random proposal of a large committee that makes decisions by weighted voting. The approach is a generalized version of James Coleman's "power of a collectivity to act". Throughout the paper it is assumed that the voters are of two kinds: a fixed (possibly empty) set of "major" (big) voters with fixed weights, and an ever-increasing number of "minor" (small) voters, whose total weight is also fixed, but where each individual's weight becomes negligible. As our main result, we obtain that asymptotically many minor voters act like a modification of the quota for the vote among major voters. The paper estimates the rate of convergence which turns out to be very high if the weight distribution among the small voters is not too skewed. The results obtained are illustrated by evaluating the decision rules for the Council of Ministers of the EU for various scenarios of EU enlargement.
\end{abstract}

\section{Introduction}

Decision rules can be characterized in terms of the way in which voting power of individuals is distributed-as represented for example by the Shapley-Shubik index (Shapley 1953) or Banzhaf (1965) measure-or by some global values. This paper is concerned with the latter, specifically one that was introduced by Coleman in his 1971 as the "power of a collectivity to act".

I wish to thank Matthew Braham, Sidartha Gordon, Maurice Koster, Moshé Machover, Guillermo Owen and two anonymous referees for helpful comments.

I. Lindner $(\bowtie)$

Department of Econometrics and Operations Research, Free University Amsterdam,

De Boelelaan 1105, 1081 HV Amsterdam, The Netherlands

e-mail: ilindner@ feweb.vu.nl 
Coleman defined the power of a collectivity to act as the a priori probability that a committee representing this collectivity will be able to pass a random bill that comes before it. The measure is simply the cardinality of winning coalitions divided by all possible coalitions. Formally, a simple voting game is a collection $\mathcal{W}$ of subsets of an assembly $N$ representing the winning coalitions; hence any $S \subset N$ with $S \notin \mathcal{W}$ is a losing coalition. The power of a collectivity to act $A$ is defined by

$$
A[\mathcal{W}]:=\frac{|\mathcal{W}|}{2^{n}}
$$

where $n=|N|$. If we read $|\mathcal{W}|$ as the number of outcomes that lead to action, then $A$ is defined as the relative number of voting outcomes leading to action. It reflects the ease with which the individual members' interests in a collective action can be translated into actual collective action. This ease is at a minimum if the collectivity operated under a decision rule in which each member has a veto-unanimity-since only the grand coalition (the total assembly) can initiate action, i.e. $A=1 / 2^{n}$. If the committee operates under simple majority rule and has an odd number of members, then exactly half of the coalitions can initiate action (for an even number of members it is slightly less than one half). The power of the collectivity is at a maximum under what Rae (1969) has called a "rule of individual initiative": where action can be initiated by a single individual, for example when s/he gives a fire alarm. In this case $A$ is obtained by $A=1-\left(1 / 2^{n}\right)$. Unless $n$ is very small $A$ will be close to one. ${ }^{1}$

Following Felsenthal and Machover (1998, p. 62) we can think of Coleman's $A$ as measuring the propensity of a committee to approve a random proposal, i.e. the complaisance of the rule $\mathcal{W}$.

The interest in Coleman's $A$ is that it allows us to say something about the ability of a collectivity that uses voting to make its decisions not only to act, but as Coleman himself said, “...to act in accord with the aims or interests of some members, but often against the aims or interests of others. Thus for a collectivity of a given size, the greater the power of the collectivity to act, the more power it has to act against the interests of some of the members" (1971, p. 277).

Interest in such a global measure as $A$ has recently emerged. Baldwin et al. (2000), Felsenthal and Machover (2001), Leech (2002a) have all made use of $A$ to evaluate the decision rules for the Council of Ministers (CM) of the EU prescribed by the Treaty of Nice for various scenarios of EU enlargement. All these studies suggest that $A$ falls significantly as the size of the voting body increases. Table 1 is taken from Felsenthal and Machover (2001) and gives the decision rules of the CM from 1958 to 1995. The greatest number of issues in EU parlance, except those concerned with the constitution of the EU itself, is decided by a rule known as qualified majority voting (QMV). From 1958 to 1995, the QMV has been a purely weighted decision rule. In a weighted voting game (WVG) each board member is assigned a non-negative number as weight and a proposed act is adopted if the combined weight of those affirming it achieves a fixed quota. Table 4 (see Appendix A) is taken from the Treaty of Nice (2001) and

\footnotetext{
1 This generally reflects the situation in which a public good, or a public bad, can be supplied by only a few members of a collectivity.
} 
Table 1 QMV weights and quota, first five periods

"Total" gives the total weight sum. "Quota" is the absolute quota; "Quota \%" indicates the relative quota in percentage terms; "min\#" gives the least number of members whose total weight equals or exceeds the (absolute) quota; " $A \%$ " is the Coleman index (1) in percentage terms

\begin{tabular}{|c|c|c|c|c|c|}
\hline Country & 1958 & 1973 & 1981 & 1986 & 1995 \\
\hline Germany & 4 & 10 & 10 & 10 & 10 \\
\hline Italy & 4 & 10 & 10 & 10 & 10 \\
\hline France & 4 & 10 & 10 & 10 & 10 \\
\hline Netherlands & 2 & 5 & 5 & 5 & 5 \\
\hline Belgium & 2 & 5 & 5 & 5 & 5 \\
\hline Luxembourg & 1 & 2 & 2 & 2 & 2 \\
\hline UK & & 10 & 10 & 10 & 10 \\
\hline Denmark & & 3 & 3 & 3 & 3 \\
\hline Ireland & & 3 & 3 & 3 & 3 \\
\hline Greece & & & 5 & 5 & 5 \\
\hline Spain & & & & 8 & 8 \\
\hline Portugal & & & & 5 & 5 \\
\hline Sweden & & & & & 4 \\
\hline Austria & & & & & 4 \\
\hline Finland & & & & & 3 \\
\hline Total & 17 & 58 & 63 & 76 & 87 \\
\hline Quota & 12 & 41 & 45 & 54 & 62 \\
\hline Quota \% & 70.59 & 70.69 & 71.43 & 71.05 & 71.26 \\
\hline $\min \#$ & 3 & 5 & 5 & 7 & 8 \\
\hline$A \%$ & 21.88 & 14.65 & 13.67 & 9.81 & 7.78 \\
\hline
\end{tabular}

represents the decision rule designed for the QMV in the EU's Council of Ministers following its enlargement to 27 member states. ${ }^{2}$

Tables 1 and 4 show an alarming decrease of $A$ from $21.88 \%$ in 1958 to $7.78 \%$ in 1995 and to $1.66 \%$ in 2004 . With each enlargement the quotient was kept in the range of $71 \pm 0.5 \%$. These numbers suggest that if the assembly keeps enlarging, while keeping the quota more or less constant, it is likely to lead to immobilism of decision making at the CM. This in turn may endanger the functioning of the whole EU. These numbers seem to suggest that as a committee expands we may have to adjust the quota in order to avoid creating an undue bias in favor of the status quo.

In (1) Coleman uses a probability distribution which is equivalent to a randomvoting model in which each member votes for or against with equal probability independently of all other members. This is not a behavioral assumption but is a method of a priori analysis. The latter models the voting system as an "abstract shell", without

\footnotetext{
2 The decision rule is not stated in the treaty in this simple form, as a weighted voting game; but it can be reduced to the form shown in Table 4. The treaty also contains a different interpretation of the agreement, which cannot be represented as a weighted game. It is thus ambiguous as to which rule applies in an enlarged 27-member CM. In the latter interpretation $A$ slightly increases to $2.025 \%$. For details see Felsenthal and Machover (2001) and Galloway (2001).
} 
taking into consideration voters' preferences, the range of issues over which a decision is taken or the degree of affinity between the voters. This abstraction seems to be necessary to evaluate the decision rule itself (for a more elaborated discussion, see Sect. 7). However, the assumption that each voter's "yes" and "no" choice is equally likely does not seem to match the observation of many real-world voting scenarios. When the nature of the analysis changes from the a priori to the actual the question is whether the pessimistic prognosis on EU's functioning still holds. In defining complaisance we take a more general route than Coleman by solely assuming that the small voters vote independently.

This paper is concerned with the asymptotic behavior of complaisance in WVGs when there are many small voters. Many economic and political organizations have systems of governance by weighted voting; examples of economic organizations are the International Monetary Fund, the World Bank, stock companies, etc. In federal political bodies the weights are usually designed to reflect the number of inhabitants of each represented state like in the case of EU's CM or the US Presidential Electoral College. However, weighted voting does not only play a central role because it is very common but also because many voting systems can be reformulated and equivalently represented as such. Taylor and Zwicker $(1992,1999)$ give a combinatorial characterization of such games.

Tables 1 and 4 show that in case of EU's CM the distribution of weights is not too skewed (in other words, the ratio of the largest weight to the smallest is not very high). However, in many large economic organizations a small group of "major" voters owns a significant number of votes usually reflecting their large capital investment. The formal setup of this paper takes care of possible different settings by assuming that the voters are of two kinds: a fixed (possibly empty) set of "major" voters with fixed weights, and an ever-increasing number of "minor" (small) voters whose total weight is also fixed but the individual weight of each minor voter becomes negligible. This approach follows Shapiro and Shapley (1978) and Dubey and Shapley (1979) who give an asymptotic analysis for the Shapley-Shubik and the Banzhaf index. We combine the analytical solution techniques of Dubey and Shapley with a general version of the central limit theorem. ${ }^{3}$ This enables us to get a closed form solution for the limit of complaisance and to estimate the rate of convergence.

Using the idea that asymptotically many minor voters act like a modification of the quota, ${ }^{4}$ this paper derives the limiting complaisance as a function of voting behavior for the vote among major voters.

The rate of convergence turns out to be very high (on an exponential rate) if the weight distribution among the small voters is not too skewed. As a rule of thumb the limit value of complaisance manifests itself at around 20 small players. The limit values are easy to compute and hence serve as a convenient approximation for large committees that apply weighted voting.

\footnotetext{
3 The approach of Banzhaf (1965) and Coleman (1971) to voting power is comparable in that both assume voters to act independently of one another. Hence some analytical proof techniques are applicable to either. 4 Dubey and Shapley (1979) use a similar argument for analyzing asymptotic properties of the Banzhaf index.
} 
Section 2 introduces the probabilistic machinery that will be used throughout the paper. Section 3 formally defines the general setup of the games. Section 4 discusses the passage of the Coleman index $A$ to the limit when the number of small voters grows to infinity. As an example Sect. 5 discusses the application of the results to the CM of the EU. Section 6 provides statements estimating the rate of convergence. Finally, Sect. 7 concludes.

\section{Preliminaries}

Let $N$ be a nonempty finite set to which we shall refer as assembly. The elements of $N$ are called voters and we shall often identify them with the integers $1,2, \ldots, n$, where $n=|N|$. A play of the voting game consists in a division in which each voter chooses one of two options (usually, "yes" and "no"). Any subset of $S \subseteq N$ is called a coalition.

Definition 2.1 A weighted voting game-briefly, WVG

$$
\left[q ; w_{1}, w_{2}, \ldots, w_{n}\right]
$$

is given by an assignment of a non-negative real weight $w_{k}$ to each voter $k \in N$, and a relative Quota $q \in(0,1)$ such that a coalition $S \subset N$ is a winning coalition iff

$$
\sum_{k \in S} w_{k} \geq q \sum_{k \in N} w_{k}
$$

The loose inequality $\geq$ in (3) may be replaced by the strict inequality $>$. In this case we shall use the notation

$$
\left\langle q ; w_{1}, w_{2}, \ldots, w_{n}\right\rangle
$$

We shall represent the choice of each minor voter $k$ by an independent random variable $X_{k}$ such that

$$
X_{k}= \begin{cases}w_{k} & \text { if } k \text { votes "yes" with probability } p_{k} \\ 0 & \text { otherwise }\end{cases}
$$

where each $p_{k}$ is bounded away from 0 and 1 .

Our main tool, borrowed from probability theory, is derived from a general version of the central limit theorem. We shall use the symbol $\Phi$ to denote the standard normal distribution. Let the expectation and variance of $X_{k}$ be denoted by

$$
\begin{gathered}
E\left[X_{k}\right]=\mu_{k}, \\
\operatorname{Var}\left[X_{k}\right]=\sigma_{k}^{2} .
\end{gathered}
$$


Further put

$$
\begin{aligned}
\left(s_{n}\right)^{2} & :=\operatorname{Var}\left[\sum_{k \leq n} X_{k}\right]=\sum_{k \leq n} \sigma_{k}^{2}, \\
S_{n} & :=\frac{1}{s_{n}} \sum_{k \leq n} X_{k}-\mu_{k},
\end{aligned}
$$

and

$$
Q_{n}:=\sum_{k \leq n} w_{k}^{2} .
$$

Theorem 2.1 In order that

$$
\lim _{n \rightarrow \infty} \sup _{x}\left|\operatorname{Prob}\left\{S_{n}<x\right\}-\Phi(x)\right|=0
$$

it is necessary and sufficient that the following condition be satisfied:

$$
\lim _{n \rightarrow \infty} \frac{w_{n}}{\sqrt{Q_{n}}}=0
$$

For a proof see Appendix B.

\section{General setup}

Consider a partition of the set of voters into two camps. The set of major voters is $L=\{1, \ldots, l\}$, where $l$ is a natural number. Note that $l=0$ takes care of the case where $L$ is empty by the general convention that $\{1, \ldots, 0\}$ is empty. Each $k \in L$ is assigned a weight $w_{k}$, and let $w_{L}=\sum_{k \in L} w_{k} \in[0,1]$ denote the combined voting weight of $L$. We shall consider a sequence of WVGs $\left\{\Gamma^{(n)}\right\}_{n \in \mathbb{N}}$ with a growing population of minor voters. In each of these games $\Gamma^{(n)}$, the set of $n$ minor voters is denoted by $M^{(n)}=\{l+1, \ldots, l+n\}$. For each $n$, these voters have weights $\alpha_{1}^{(n)}, \ldots, \alpha_{n}^{(n)}$, which sum up to $\alpha=1-w_{L}>0$.

Formally, the WVG $\Gamma^{(n)}$ is described by the tuple

$$
\Gamma^{(n)}=\left[q ; w_{1}, \ldots, w_{l}, \alpha_{1}^{(n)}, \ldots, \alpha_{n}^{(n)}\right],
$$

where $q \in(0,1]$ is the relative quota.

Put $\alpha_{\max }^{(n)}:=\max _{k \leq n} \alpha_{k}^{(n)}$ and $Q^{(n)}:=\sum_{k \leq n}\left[\alpha_{k}^{(n)}\right]^{2}$. Let $\left\{\Gamma^{(n)}\right\}_{n \in \mathbb{N}}$ evolve in such a way that

$$
\lim _{n \rightarrow \infty} \alpha_{\max }^{(n)} / \sqrt{Q^{(n)}}=0
$$

which ensures $\alpha_{\max }^{(n)} \rightarrow 0$ as $n \rightarrow \infty .^{5}$

\footnotetext{
${ }^{5}$ However, it can be shown that $Q^{(n)}$ tends to zero so that condition (9) is stronger than $\alpha_{\max }^{(n)} \rightarrow 0$ : it holds that $\sum_{k}\left(\alpha_{k}\right)^{2} \leq \sum_{k} \alpha_{k} \alpha_{\max }^{(n)} \leq \alpha_{\max }^{(n)} \sum_{k} \alpha_{k}=\alpha_{\max }^{(n)} \alpha$. Now, since $\alpha$ is fixed, but $\alpha_{\max }^{(n)} \rightarrow 0$, it follows that $\sum_{k}\left(\alpha_{k}\right)^{2}$ also goes to 0 .
} 
We do not impose any behavioral assumption on the major voters, however, for the minor voters we assume:

Assumption 3.1 Each minor voter $k$ votes "yes" independently with probability $p_{k}$ bounded away from 0 and 1 .

Throughout our analysis we shall interpret the weight sum of a random coalition of minor voters as a sum of random variables which are independent according to Assumption 3.1. This interpretation allows to derive the limit distribution by means of the normal distribution as depicted in (6) of Theorem 2.1. Condition (9) ensures that (7) holds.

In the following let $\alpha^{(n)}(S)=\sum_{k \in S} \alpha_{k}^{(n)}$ denote the random weight sum of the affirming minor voters $S$, where $S$ is a random subset of $M^{(n)}$. Let the expectation of $\alpha^{(n)}(S)$ be denoted by

$$
\mu^{(n)}:=E\left[\alpha^{(n)}(S)\right]=\sum_{k \leq n} p_{k} \alpha_{k}^{(n)} .
$$

Note that in the a priori case we have $\mu^{(n)}=\alpha / 2$ for all $n$. For general $p_{k}$ we assume that $\mu^{(n)}$ converges to the limit $\mu$ with order $o\left(\sqrt{Q^{(n)}}\right)$.

\section{Complaisance in WVG}

Let $A[\Gamma]$ denote the complaisance of any game $\Gamma$. Note that with Assumption 3.1 we take a more general route than Coleman's $A$ as defined in (1). We shall refer to the latter as a priori complaisance.

Assumption 3.1 suggests that in the limit the continuous "ocean" of minor voters would be divided such that the affirming voters represent a fraction $\mu$ of the total minor weight sum $\alpha .{ }^{6}$ Formally, this suggests to focus on the following games

$$
\begin{aligned}
& \Gamma_{0}=\left[q-\mu ; w_{1}, \ldots, w_{l}\right], \\
& \Gamma_{0}^{\prime}=\left\langle q-\mu ; w_{1}, \ldots, w_{l}\right\rangle .
\end{aligned}
$$

Let $\mathcal{B}_{l}=\left[w_{L} ; w_{1}, w_{2}, \ldots, w_{l}\right]$ denote the unanimity game among the major voters in which each voter has a veto. Let $\mathcal{B}_{l}^{*}=\left\langle 0 ; w_{1}, w_{2}, \ldots, w_{l}\right\rangle$ represent the special case where the major voters operate under what Rae (1969) has called a "rule of individual initiative": action can be initiated by any single individual.

Put

$$
\begin{aligned}
\mathcal{R} & :=\{q \mid 0<q<1\}, \\
\mathcal{J} & :=\left\{q \mid \mu<q<w_{L}+\mu\right\} .
\end{aligned}
$$

\footnotetext{
${ }^{6}$ We owe this insight to Dubey and Shapley (1979) who use a similar argument for an asymptotic analysis of the Banzhaf index. Theorem 4.1 of the present paper can be seen as an analogue to their Theorem 8 , p. 116 .
} 


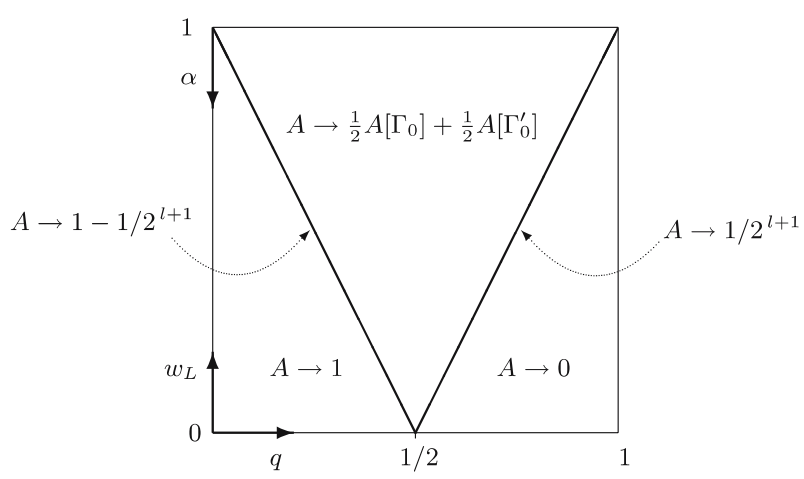

Fig. 1 Limit scenario for a priori complaisance in WVGs

Theorem 4.1 In the sequence of games $\left\{\Gamma^{(n)}\right\}_{n \in \mathbb{N}}$ we have

$$
\lim _{n \rightarrow \infty} A\left[\Gamma^{(n)}\right]=\frac{1}{2} A\left[\Gamma_{0}\right]+\frac{1}{2} A\left[\Gamma_{0}^{\prime}\right] \quad \text { if } q \in \mathcal{J}
$$

For other values of $q$, the right-hand side of (14) simplifies to

$$
\lim _{n \rightarrow \infty} A\left[\Gamma^{(n)}\right]= \begin{cases}1 & \text { if } q<\mu, \\ \frac{1}{2}\left(1+A\left[\mathcal{B}_{l}^{*}\right]\right) & \text { if } q=\mu, \\ \frac{1}{2} A\left[\mathcal{B}_{l}\right] & \text { if } q=w_{L}+\mu, \\ 0 & \text { if } q>w_{L}+\mu .\end{cases}
$$

For a proof see Appendix C.

Figure 1 illustrates the result for a priori complaisance and hence $\mu=\alpha / 2$. The asymptotic value of $A$ is determined by the relation of the quota $q$ to the total major weight $w_{L}$. At the interior region $\mathcal{J}=\left\{q \mid \alpha / 2<q<w_{L}+\alpha / 2\right\}$ the limit follows as the arithmetical mean of complaisance of finite major games as defined in (11). Let the closure of $\mathcal{J}$ be denoted by $\overline{\mathcal{J}}$. In the domain $\mathcal{R}-\overline{\mathcal{J}}$ the influence of the major voters is "destroyed": in the limit we have a combined voting weight of exactly $\alpha / 2$ affirming minor voters such that $q<\alpha / 2$, the left triangle, always ensures the passage of a proposal. The opposite holds for $w_{L}+\alpha / 2<q$, the right triangle, respectively. Here, even with all major voters affirming the quota $q$ is too high for any motion to pass.

For general $\left\{p_{k}\right\}_{k=1}^{\infty}$, in particular for general $\mu$, the scenario as depicted in Fig. 1 stays topologically the same, however, the interior region $\mathcal{J}$ is distorted. The reason is a shift of the triangle's lower point at the $w_{L}=0$ axis to the right for $\mu>\alpha / 2$, to the left for $\mu<\alpha / 2$, respectively, as illustrated in Fig. 2. 


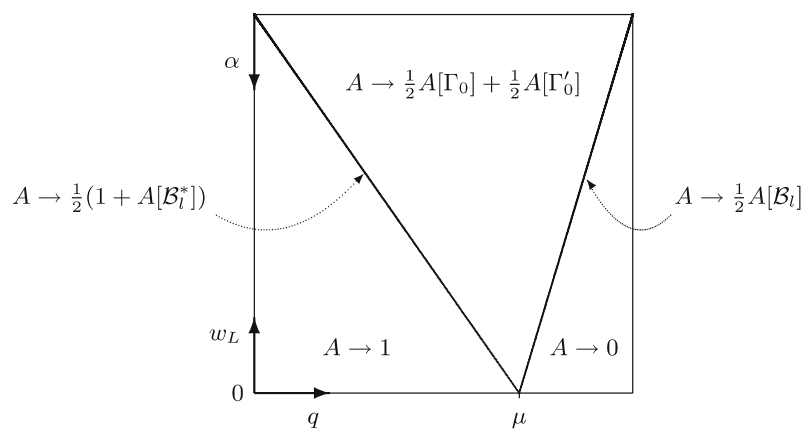

Fig. 2 Limit scenario for general complaisance

\section{An example: the EU council of ministers}

This section applies the results on complaisance to the expansion of EU's CM since 1958. The substantial property of the process was that with each enlargement of the EU the maximal normalized voting weight decreased while the relative quota was kept more or less constant at $71 \%$ as indicated by Tables 1 and 4 . We shall therefore interpret the enlargement of the CM as a sequence of WVGs with an empty set of major voters. In formal terms, we put

$$
\Gamma^{(n)}=\left[q ; \alpha_{1}, \ldots, \alpha_{n}\right]
$$

with $L=\emptyset$. We shall identify the five scenarios from 1958 to 1995 and the QMV following its prospective enlargement to 27 as sequence elements $\Gamma^{(6)}, \Gamma^{(9)}, \ldots, \Gamma^{(15)}$, $\Gamma^{(27)}$, where the index denotes the size of the Council. The second row of Table 2 suggests that these games can be interpreted as elements of a sequence satisfying condition (9).

The limit scenario for WVGs with no major voters is depicted by the horizontal axis $w_{L}=0$ and hence $\alpha=1$ in Figs. 1 and 2. Theorem 4.1 provides for games with an empty set of major voters

$$
\lim _{n \rightarrow \infty} A\left[\Gamma^{(n)}\right]= \begin{cases}1 & \text { if } q<\mu, \\ 1 / 2 & \text { if } q=\mu, \\ 0 & \text { if } q>\mu\end{cases}
$$

where $\mu=1 / 2$ for a priori complaisance.

Table 2 Evolution of the CM

\begin{tabular}{lcccccc}
\hline & $\Gamma^{(6)}$ & $\Gamma^{(9)}$ & $\Gamma^{(10)}$ & $\Gamma^{(12)}$ & $\Gamma^{(15)}$ & $\Gamma^{(27)}$ \\
\hline$\alpha_{\max }^{(n)}$ & 0.2353 & 0.1724 & 0.1587 & 0.1316 & 0.1149 & 0.0852 \\
$\alpha_{\max }^{(n)} / \sqrt{Q^{(n)}}$ & 0.5298 & 0.4603 & 0.4486 & 0.4131 & 0.3994 & 0.3627 \\
\hline
\end{tabular}




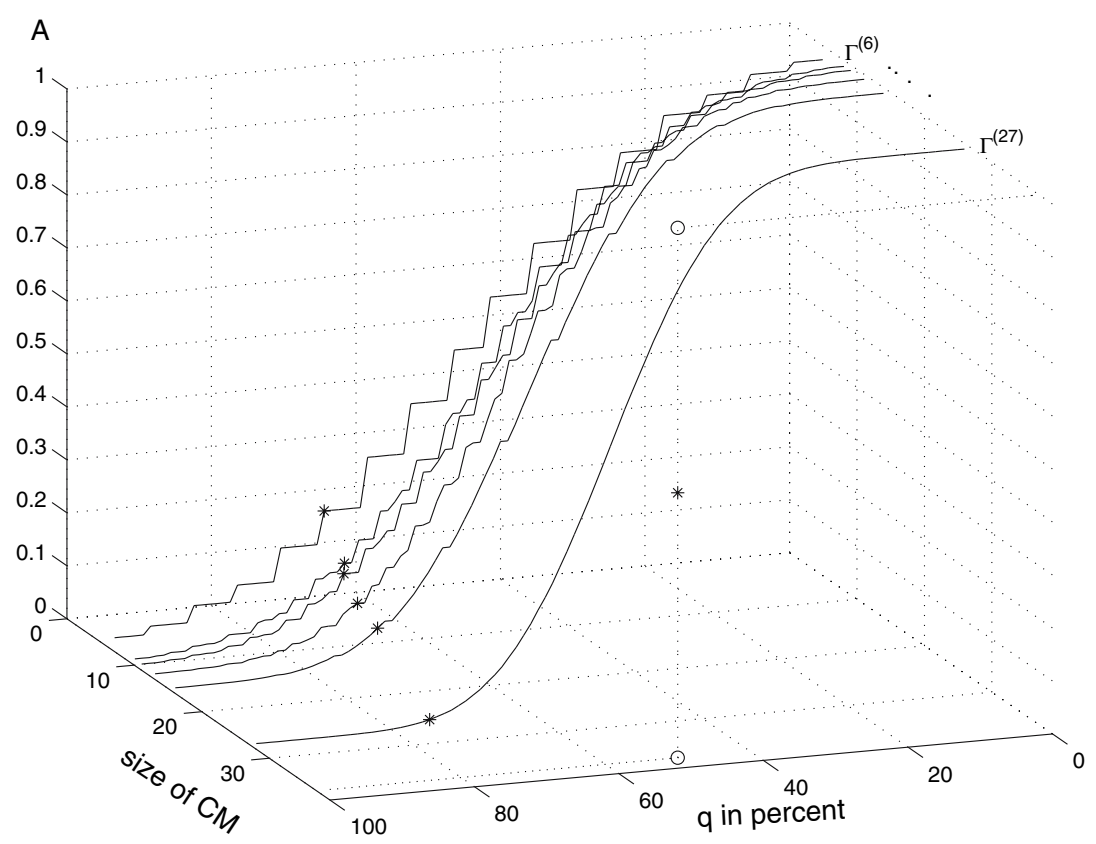

Fig. 3 Complaisance in EU council of ministers

Figure 3 illustrates a priori complaisance $A\left[\Gamma^{(n)}\right]$ for the six scenarios $\Gamma^{(6)}, \Gamma^{(9)}$, $\ldots, \Gamma^{(27)}$ as a function of $q \in(0,1)$. The points marked with " $*$ " are the corresponding realizations of $q$ in $\mathrm{CM}$ as given by Tables 1 and 4 . The step function in the front indicates (in dashed lines) the limit values with increasing number of voters. As a reference scenario, Fig. 4 provides the same picture for the symmetric weight distribution "one person one vote" with $\alpha_{k}^{(n)}=\alpha / n$ for all $k=1, \ldots, n$ which are qualitatively similar to the WVGs of the CM.

In all scenarios we observe that convergence tends to be relatively quick for $q \neq 0.5$. For a detailed convergence analysis see Sect. 6 . Higher the convergence rate, the closer $q$ gets to the boundaries $q=0$ and $q=1$ as indicated by Lemmas 6.1 and 6.2 in the following section.

Interestingly Figs. 3 and 4 identify another indicator for the dramatic decrease of a priori $A$ in the process of enlarging EU: both exhibit a high sensitivity to changes in $q$ especially for low values of $n$. It turns out that this sensitivity has a large impact. The first three columns of Table 1 indicate that the decrease of $A$ is due to a slight increase in the quota from $70.59 \%$ in $\Gamma^{(6)}$ to $70.69 \%$ in $\Gamma^{(9)}$ and $71.43 \%$ in $\Gamma^{(10)}$. Row $A_{71}$ in Table 3 gives the corresponding values if the quota $q$ had been kept at constant $71 \%$ which represents the arithmetic mean of the quotas from 1958 to 1995 . In this case the first scenario would have started with a lower value $A\left[\Gamma^{(6)}\right]$ and hence the difference in comparison to the subsequent scenario would have been less significant. In fact, with a fixed quota $q=0.71$ a priori complaisance would have slightly increased from 1973 to 1981. 


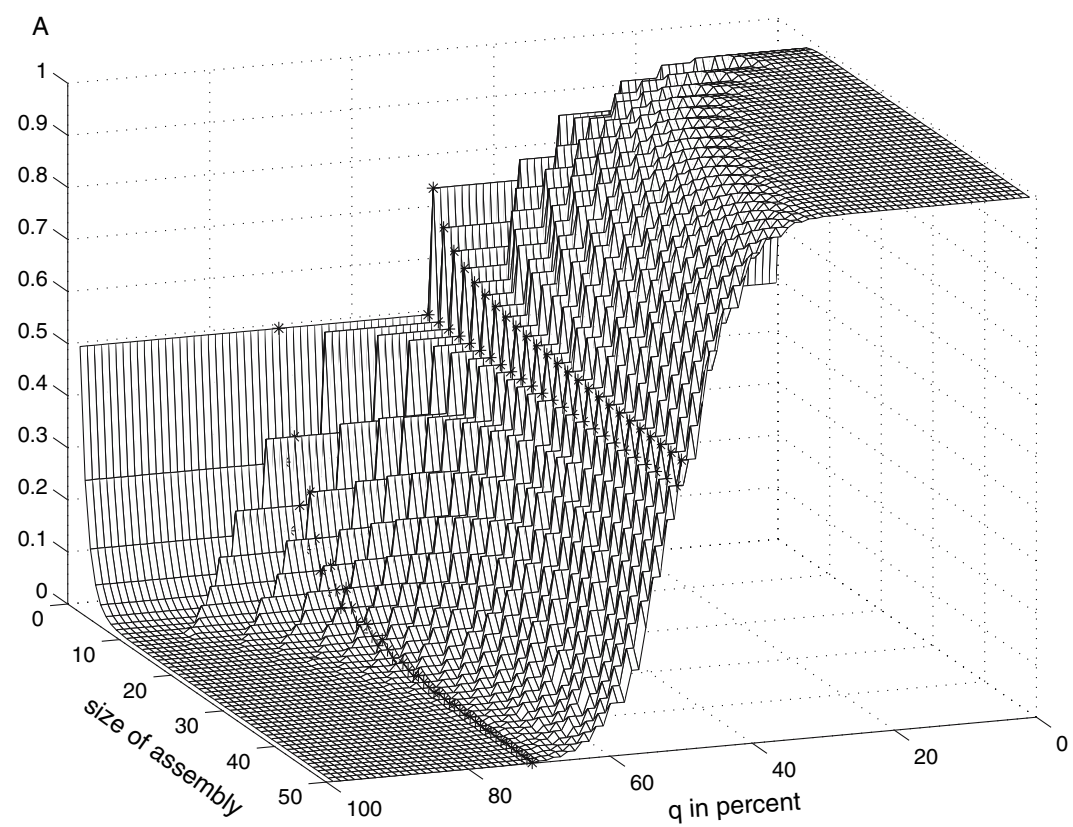

Fig. 4 One person one vote

Table 3 Complaisance of QMV with fixed $71 \%$, first five periods

\begin{tabular}{llllrr}
\hline Country & 1958 & 1973 & 1981 & 1986 & 1995 \\
\hline Quota \% & 70.59 & 70.69 & 71.43 & 71.05 & 71.26 \\
$A \%$ & 21.88 & 14.65 & 13.67 & 9.81 & 7.78 \\
$A_{71} \%$ & 15.62 & 13.09 & 13.67 & 9.81 & 7.78 \\
\hline
\end{tabular}

\section{Convergence characteristics}

Let $T$ denote a random subset of $L, S$ be a random subset of $M^{(n)}$, respectively. For any $B \subset L$ put $w_{B}:=\sum_{k \in B} w_{k}$. Disentangling the vote among major and minor voters complaisance can be represented as

$$
A\left[\Gamma^{(n)}\right]=\sum_{B \subset L} \operatorname{Prob}\{T=B\} \operatorname{Prob}\left\{\alpha^{n}(S) \geq q-w_{B}\right\} .
$$

Hence complaisance can be derived as finite sums of (weighted) terms

$$
\operatorname{Prob}\left\{\alpha^{(n)}(S) \geq z\right\}, \quad z \in \mathbb{R},
$$

determining the convergence behavior which in turn hinges on the relation of $z$ to the mean value $\mu^{(n)}$. We shall see that the rate of convergence is high if $z \neq \mu^{(n)}$ and if the distribution of the minor votes is reasonably smooth. 
Lemma 6.1 In the sequence of games $\left\{\Gamma^{(n)}\right\}_{n \in \mathbb{N}}$ defined by (8) we have under Assumption 3.1

$$
\operatorname{Prob}\left\{\left|\alpha^{(n)}(S)-\mu^{(n)}\right|>\varepsilon\right\} \leq \exp \left[-\left(\frac{\varepsilon}{2 \sqrt{n} \alpha_{\max }^{(n)}}\right)^{2}\right]
$$

For a proof see Appendix D.

In the following consideration we assume for simplicity $\mu^{(n)}=\mu$ for all $n \in \mathbb{N}$. The statements are easily extendable for more general $\mu^{(n)}$ for sufficiently large $n$ (such that $\mu^{(n)}$ is sufficiently close to $\mu$ ). We shall focus on weight distributions of the major voters and the quota $q$ such that $z \neq \mu$ always holds. For a "tail" estimation we shall exclude the set $P$ given by

$$
P:=\left\{q \mid q-w_{B}=\mu \text { for some } B \subseteq L\right\} .
$$

Put

$$
\lambda:=\min _{B \subset L}\left\{\left|q-w_{B}-\mu\right|\right\}
$$

Corollary 6.1 In the sequence of games $\left\{\Gamma^{(n)}\right\}_{n \in \mathbb{N}}$ defined by (8) we have under Assumption 3.1 and for $q \notin P$

$$
\left|A\left[\Gamma^{(n)}\right]-\tilde{A}\right| \leq \exp \left[-\left(\frac{\lambda}{2 \sqrt{n} \alpha_{\max }^{(n)}}\right)^{2}\right]
$$

where $\tilde{A}=\lim _{n \rightarrow \infty} A\left[\Gamma^{(n)}\right]$.

From Corollary 6.1 it follows that the rate of converges hinges on $\sqrt{n} \alpha_{\max }^{(n)}$. If the weight distribution among the minor voters is sufficiently smooth so that $\sqrt{n} \alpha_{\max }^{(n)}$ tends to zero sufficiently fast ${ }^{7}$ we can expect high rates of convergence.

Corollary 6.2 Under the conditions of Corollary 6.1 we have for $\alpha_{\max }^{(n)} \leq \gamma / n$

$$
\left|A\left[\Gamma^{(n)}\right]-\tilde{A}\right| \leq \exp \left[-n\left(\frac{\lambda}{2 \gamma}\right)^{2}\right]
$$

Section 5 discussed a priori complaisance in absence of major voters. Figures 3 and 4 illustrate that if the number of voters increases while at the same time the quota is pegged at a constant percentage, the scenario is qualitatively comparable to the "one person one vote" situation (the symmetric case). However, there is a distortion effect due to unequal weight distribution. The following theorem provides a statement for $l=0$, considering the ratio $\alpha_{\max }^{(n)} / \alpha_{\min }^{(n)}$.

\footnotetext{
7 Note that the condition $\sqrt{n} \alpha_{\max }^{(n)} \rightarrow 0$ is stricter than (9).
} 
Lemma 6.2 If every subset $S \subset M^{(n)}$ is equally probable then for any $\varepsilon>0$,

$$
\operatorname{Prob}\left\{\alpha^{(n)}(S)>\alpha / 2+\varepsilon\right\} \leq \exp \left[-\frac{8 n \varepsilon^{2} \theta}{\alpha^{2}(1+\theta)^{2}}\right],
$$

where $\theta$ denotes $\alpha_{\max }^{(n)} / \alpha_{\min }^{(n)}$.

For a proof see Hoeffding (1963).

\section{Discussion and concluding remarks}

In his seminal paper Coleman (1971) introduced a priori complaisance which is based on the assumption that each "yes" and "no" choice is equally likely. Contrary to actual (a posteriori) analysis, it models the voting system as an "abstract shell", without taking into consideration voters' preferences, the range of issues over which a decision is taken or the degree of affinity between the voters. This abstraction seems to be necessary to evaluate the decision rule itself. Roth (1988, p. 9) puts it this way:

Analyzing voting rules that are modeled as simple games abstracts away from the particular personalities and political interests present in particular voting environments, but this abstraction is what makes the analysis focus on the rules themselves rather than on other aspects of the political environment. This kind of analysis seems to be just what is needed to analyze the voting rules in a new constitution, for example, long before the specific issues to be voted on arise or the specific factions and personalities that will be involved can be identified.

It is tempting to question a pessimistic prognosis based solely on a priori complaisance. The assumption that voters act independently and vote at random "yes" or "no" with equal probability does not match the observation of many real-world voting scenarios, as for example the CM: when it comes to voting the affirmative votes usually represent a majority. Hence when changing the nature of the analysis from the a priori to the actual this suggests to modify the a priori probabilistic-voting assumption in order to introduce more realism. However, care must be taken when leaving the a priori ground since recording voting behavior for or against a proposal can be misleading. The outcome of a vote is usually the result of a foregoing bargaining process. Before the formal vote is taken there is usually a whole series of shadow or straw divisions- which comes to a halt when a majority can be expected. ${ }^{8}$ In that sense, a priori complaisance can be thought of as measuring the barrier that members of a committee have to overtake via negotiations and bargaining in order to approve a given proposal (for models of bargaining in committees see e.g. Laruelle and Valenciano 2006). An increase of this barrier is usually reflected by a long pre-vote period and therefore imparts a considerable bias in favor of the status quo.

The present analysis takes a general route-in probabilistic terms-by solely assuming that the minor voters vote independently which covers the a priori approach

\footnotetext{
8 Also, many committees as, e.g., the CM seem to publish only positive outcomes, i.e., when acts have been adopted.
} 
as a special case. However, when we are concerned with a posteriori or actual (rather than a priori) voting power, surely also the assumption that all (or indeed any) voters are independent cannot be taken for granted. What would be the effect on complaisance in large WVGs when voters' choices are correlated? This is still an open question. Addressing it, however, would require different analytical tools from those used in the present paper and is therefore left for future investigations.

Under the independence assumption, asymptotically many minor voters act like a modification of the quota for the vote among major voters. Absence of major voters reflects the scenario when the distribution of the voting weights of a large committee is not too skewed (in other words, if the ratio of the largest weight to the smallest is not very high). Examples are the US Presidential Electoral College or the CM of the EU. Our analysis covers absence of major voters as a special case. Here, we have shown that if the quota is pegged at a constant percentage which is greater than the expected affirming minor votes, then as the number of voters increases complaisance falls close to zero very fast. This could pose a serious threat to the functioning of the EU.

When it comes to designing the constitution of a future decision-making body, there is a near-consensus that we must take an aprioristic stance. Even if information on specific interests, preferences and affinities of the voters is presently available there is, in general, very little reliable information about future developments. This holds in particular for the $\mathrm{CM}$ where the political orientation of its members changes every few years. For the CM, the lesson would be that to maintain a desired level of a priori complaisance, the quota need to be moved closer to $50 \%$ with each enlargement. Instead, it is raised in the Treaty of Nice to $74.66 \%$. Hence the main conclusion is that decision making within the $\mathrm{CM}$ of the EU is likely to get more rigid with every enlargement which endangers the effectiveness of the Council.

The reason for the high quota of the CM since its origin in 1958 was presumably the concern of the individual member countries with their own blocking powers (Leech 2002a). However, while choosing a high level for the threshold has the advantage of protecting (the large) countries against being outvoted too easily, it also restricts the ability of all members to get their own proposals accepted. Felsenthal and Machover (2000) offer the explanation that the politicians and officials who decided on the weights and quota might have been mislead by the following naive and fallacious assumption: by pegging the quota at about $71 \%$ of the total weight, they would be keeping complaisance more or less constant as with each enlargement of the EU, the same proportion of the total weight-about $29 \%$ - would be needed to block a resolution.

This misconception shows that the extent of common acceptance and application of measures of complaisance to real-life voting design is still modest. A major limiting factor is presumably that the computation is not straightforward. The standard approach for evaluating WVGs is a method using generating functions (see Mann and Shapley 1960) ${ }^{9}$. Unfortunately, this method is not without limitation due to space complexity in both integer size and array dimension. For example, Leech (2002a) who examines voting power for the weighted rules of the IMF, uses approximations

\footnotetext{
9 The key computational idea goes back to David G. Cantor who suggested it to the authors, following a lecture at Princeton University in October 1960.
} 
to cope with comparable numerical challenges. The present paper shows that with an increasing number of voters complaisance converges very fast (on an exponential rate) if the weight distribution among the small voters is not too skewed. As a rule of thumb the limit values manifests itself at around 20 small players. These values are easy to compute and hence serve as a convenient approximation for large committees that apply weighted voting.

\section{Appendix A. WVG from the Treaty of Nice}

Table 4 QMV under $\mathcal{N}_{27}$

\begin{tabular}{|c|c|c|}
\hline Country & $\begin{array}{l}(1) \\
w\end{array}$ & $\begin{array}{l}(2) \\
\bar{w}(\%)\end{array}$ \\
\hline Germany & 118 & 8.5199 \\
\hline UK & 117 & 8.4477 \\
\hline France & 117 & 8.4477 \\
\hline Italy & 117 & 8.4477 \\
\hline Spain & 108 & 7.7978 \\
\hline Poland & 108 & 7.7978 \\
\hline Romania & 56 & 4.0433 \\
\hline Netherlands & 52 & 3.7545 \\
\hline Greece & 48 & 3.4657 \\
\hline Czech Republic & 48 & 3.4657 \\
\hline Belgium & 48 & 3.4657 \\
\hline Hungary & 48 & 3.4657 \\
\hline Portugal & 48 & 3.4657 \\
\hline Sweden & 40 & 2.8881 \\
\hline Bulgaria & 40 & 2.8881 \\
\hline Austria & 40 & 2.8881 \\
\hline Slovakia & 28 & 2.0217 \\
\hline Denmark & 28 & 2.0217 \\
\hline Finland & 28 & 2.0217 \\
\hline Ireland & 28 & 2.0217 \\
\hline Lithuania & 28 & 2.0217 \\
\hline Latvia & 16 & 1.1552 \\
\hline Slovenia & 16 & 1.1552 \\
\hline Estonia & 16 & 1.1552 \\
\hline Cyprus & 16 & 1.1552 \\
\hline Luxembourg & 16 & 1.1552 \\
\hline Malta & 12 & 0.8664 \\
\hline Total & 1,385 & 100.0001 \\
\hline
\end{tabular}




\section{Appendix B. Proof of Theorem 2.1}

Theorem B.1 (Lindeberg-Feller)

Let $\left\{X_{k}\right\}_{k=1}^{\infty}$ be a sequence of independent random variables, at least one of which has a non-degenerate distribution. Let the distribution of $X_{k}$ be denoted by $F_{k}$, its expectation by $E\left[X_{k}\right]=\mu_{k}$ and assume its variance $\operatorname{Var}\left[X_{k}\right]=\sigma_{k}^{2}$ to be finite. Further put

$$
\begin{aligned}
\left(s_{n}\right)^{2} & :=\operatorname{Var}\left[\sum_{k \leq n} X_{k}\right]=\sum_{k \leq n} \sigma_{k}^{2}, \\
S_{n} & :=\frac{1}{s_{n}} \sum_{k \leq n} X_{k}-\mu_{k} .
\end{aligned}
$$

In order that

$$
\lim _{n \rightarrow \infty} \max _{k \leq n} \frac{\sigma_{k}}{s_{n}}=0
$$

and

$$
\lim _{n \rightarrow \infty} \sup _{x}\left|\operatorname{Prob}\left\{S_{n}<x\right\}-\Phi(x)\right|=0
$$

it is necessary and sufficient that the following condition (the Lindeberg condition) be satisfied:

$$
\lim _{n \rightarrow \infty} L_{n}(\varepsilon)=0
$$

with

$$
\begin{aligned}
L_{n}(\varepsilon) & :=s_{n}^{-1} \sum_{k \leq n} E\left[\left(X_{k}-\mu_{k}\right)^{2} ;\left|X_{k}-\mu_{k}\right| \geq \varepsilon s_{n}\right] \\
& =s_{n}^{-1} \sum_{k \leq n} \int_{\left\{\left|x-\mu_{k}\right| \geq \varepsilon s_{n}\right\}}\left(x-\mu_{k}\right)^{2} d F_{k}(x)
\end{aligned}
$$

for every fixed $\varepsilon>0$.

For a proof see, e.g., Petrov (1975), pp. 100-101.

Lemma B.1 The sequence $\left\{X_{k}\right\}_{k=1}^{\infty}$ as given by (5) satisfies the Lindeberg condition (26) iff

$$
\lim _{n \rightarrow \infty} \frac{w_{n}}{\sqrt{Q_{n}}}=0 .
$$

Proof For each $k$ follows

$$
\begin{aligned}
E\left[X_{k}\right] & =p_{k} w_{k}, \\
\operatorname{Var}\left[X_{k}\right] & =p_{k}\left(1-p_{k}\right) w_{k}^{2}, \\
s_{n}^{2} & =\sum_{k \leq n} p_{k}\left(1-p_{k}\right) w_{k}^{2} .
\end{aligned}
$$


Since any $p_{k}$ is bounded away from 0 and 1 there exists a $\delta=\min _{k \leq n}\left\{p_{k}\left(1-p_{k}\right)\right\}>0$. For $s_{n}$ follows

$$
\delta Q_{n} \leq s_{n}^{2} \leq \frac{1}{4} Q_{n}
$$

Now suppose the Lindeberg condition (26) is satisfied. Then by Theorem B.1 we have (24), from which (28) follows at once in view of (30), (31) and (32).

Conversely, suppose that (28) holds. We now show that

$$
\lim _{n \rightarrow \infty} \max _{k \leq n} \frac{w_{k}}{\sqrt{Q_{n}}}=0 .
$$

For any $\varepsilon>0$ fix $n^{\prime}$ so large that $w_{k} / \sqrt{Q_{k}}<\varepsilon$ for all $k>n^{\prime}$. Thus, for all $n>n^{\prime}$ we have

$$
\frac{w_{k}}{\sqrt{Q_{n}}} \leq \frac{w_{k}}{\sqrt{Q_{k}}}<\varepsilon \quad \text { for } k=n^{\prime}+1, \ldots, n
$$

Thus (33) holds. Now observe that for every $k$, the integral in (27) follows as

$$
\int_{\left|x-p_{k} w_{k}\right|>\varepsilon s_{n}}\left(x-p_{k} w_{k}\right)^{2} d F_{k}(x) .
$$

But from $\left|x-p_{k} w_{k}\right|=\left|y-p_{k}\right| w_{k}$ for all $y \in[0,1]$ and from (32) and (33) it follows that, for any given $\varepsilon>0$, if $n$ is sufficiently large, then

$$
\left|y-p_{k}\right| w_{k}<\varepsilon s_{n}
$$

for all $y \in[0,1]$ and all $k \leq n$. That implies the integral (34) vanishes for all $k \leq n$. Hence (26) holds.

\section{Appendix C. Proof of Theorem 4.1}

We represent the vote of each minor voter $k \in M^{(n)}$ as the random variable

$$
X_{k}^{(n)}= \begin{cases}\alpha_{k}^{(n)} & \text { if } k \text { votes "yes" } \\ 0 & \text { otherwise. }\end{cases}
$$

The expectation $\mu^{(n)}$ and standard deviation $s^{(n)}$ of $\sum_{k \leq n} X_{k}^{(n)}$ is given by

$$
\begin{aligned}
\mu^{(n)} & =\sum_{k \leq n} E\left[X_{k}^{(n)}\right]=\sum_{k \leq n} p_{k} \alpha_{k}^{(n)}, \\
\left(s^{(n)}\right)^{2} & =\sum_{k \leq n} p_{k}\left(1-p_{k}\right)\left(\alpha_{k}^{(n)}\right)^{2} .
\end{aligned}
$$


Since the $p_{k}$ are bounded away from zero and one there exists a $\delta>0$ such that

$$
s^{(n)} \geq \delta \sqrt{Q^{(n)}} .
$$

For the proof of Theorem 4.1 we shall use the following Lemma.

Lemma C.1 Let $0 \leq z \leq \alpha$ and choose a subset $S \subseteq M^{(n)}$ at random. For the sequence of games given by (8) and (9) we have

$$
\lim _{n \rightarrow \infty} \operatorname{Prob}\left\{\alpha^{(n)}(S) \geq z\right\}= \begin{cases}1 & \text { if } z<\mu \\ 1 / 2 & \text { if } z=\mu \\ 0 & \text { if } z>\mu\end{cases}
$$

Proof From Theorem 2.1 follows

$$
\lim _{n \rightarrow \infty} \operatorname{Prob}\left\{\alpha^{(n)}(S)<z\right\}=\lim _{n \rightarrow \infty} \Phi\left(\frac{z-\mu^{(n)}}{s^{(n)}}\right)
$$

With increasing $n$ the standard deviation $s^{(n)}$ tends to zero which provides (39) for $z \neq q$. For $z=\mu$ note that the argument of $\Phi$ is a fraction of two zero sequences. However, in Sect. 3 we assumed that $\mu^{(n)}$ converges with order $o\left(\sqrt{Q^{(n)}}\right)$ such that from (38) follows that this fraction converges to zero.

For any $B \subset L$ put $w_{B}:=\sum_{k \in B} w_{k}$. Let $T \subset L$ denote a random subset of $L$.

$$
A\left[\Gamma^{(n)}\right]=\sum_{B \subset L} \operatorname{Prob}\{T=B\} \operatorname{Prob}\left\{\alpha^{n}(S) \geq q-w_{B}\right\}
$$

Proof of Theorem 4.1 From Lemma C.1 follows

$$
\operatorname{Prob}\left\{\alpha^{(n)}(S) \geq q-w_{B}\right\} \rightarrow\left\{\begin{array}{lr}
0 & w_{B}<q-\mu \\
1 / 2 & \text { if } w_{B}=q-\mu \\
1 & w_{B}>q-\mu
\end{array}\right.
$$

For $q \in \mathcal{J}$ the games $\Gamma_{0}$ and $\Gamma_{0}^{\prime}$ are well defined, and for any $B \subseteq L$ for which the limit of $\operatorname{Prob}\left\{\alpha^{(n)}(S) \geq q-w_{B}\right\}$ is 1 we have that $B$ is a winning coalition in both $\Gamma_{0}$ and $\Gamma_{0}^{\prime}$. If the limit is $1 / 2$ the coalition $B$ is winning in $\Gamma_{0}$ but not $\Gamma_{0}^{\prime}$. This yields for (41)

$$
\begin{aligned}
\lim _{n \rightarrow \infty} A\left[\Gamma^{(n)}\right] & =\sum_{w_{B}>q-\mu} \operatorname{Prob}\{T=B\}+\frac{1}{2} \sum_{w_{B}=q-\mu} \operatorname{Prob}\{T=B\} \\
& =\frac{1}{2} \sum_{w_{B}>q-\mu} \operatorname{Prob}\{T=B\}+\frac{1}{2} \sum_{w_{B} \geq q-\mu} \operatorname{Prob}\{T=B\} \\
& =\frac{1}{2} A\left[\Gamma_{0}\right]+\frac{1}{2} A\left[\Gamma_{0}^{\prime}\right],
\end{aligned}
$$

and hence (14). 
To see (15) note that from $q<\mu$ follows that the third condition in (42) is fulfilled for any $B \subseteq L$ and hence Prob $\left\{\alpha^{(n)}(S) \geq q-w_{B}\right\} \rightarrow 1$ for all $B \subseteq L$.

The equality $q=\mu$ implies $\operatorname{Prob}\left\{\alpha^{(n)}(S) \geq q-w_{B}\right\} \rightarrow 1 / 2$ for $B=\emptyset$ and 1 otherwise which yields

$$
\begin{aligned}
\lim _{n \rightarrow \infty} A\left[\Gamma^{(n)}\right] & =\sum_{B \neq \emptyset} \operatorname{Prob}\{T=B\}+\frac{1}{2} \operatorname{Prob}\{T=\emptyset\} \\
& =A\left[\mathcal{B}_{l}^{*}\right]+\frac{1}{2}\left(1-A\left[\mathcal{B}_{l}^{*}\right]\right)=\frac{1}{2}\left(1+A\left[\mathcal{B}_{l}^{*}\right]\right) .
\end{aligned}
$$

If $q=w_{L}+\mu$ then $\operatorname{Prob}\left\{\alpha^{(n)}(S) \geq q-w_{B}\right\} \rightarrow 1 / 2$ for $B=L$ and 0 otherwise.

Finally, from $q>w_{L}+\mu$ follows that $\operatorname{Prob}\left\{\alpha^{(n)}(S) \geq q-w_{B}\right\} \rightarrow 0$ for any $B \subseteq L$.

\section{Appendix D. Proof of Lemma 6.1}

We shall use the following Lemma of Kemperman (1964).

Lemma D.1 Let $\left\{Z_{k}\right\}_{k=1}^{\infty}$ be a sequence of independent real-valued random variables such that $\left|Z_{k}\right| \leq 1$ for all $k$. Let further $\left\{c_{k}\right\}_{k=1}^{\infty}$ be a sequence of real constants such that

$$
s^{2}=\sum_{k=0}^{\infty} c_{k}^{2}<\infty
$$

Then

$$
\tilde{Z}=\sum_{k=0}^{\infty} c_{k}\left(Z_{k}-\mu_{k}\right)
$$

with $\mu_{k}=E\left[Z_{k}\right]$, satisfies

$$
\operatorname{Prob}\{\tilde{Z}>\delta s\} \leq \exp \left[-\frac{\delta^{2}}{2}\right], \quad \operatorname{Prob}\{\tilde{Z}<-\delta s\} \leq \exp \left[-\frac{\delta^{2}}{2}\right]
$$

for each number $\delta>0$.

Proof of Lemma $6.1{ }^{10}$ For a fixed $n$ let

$$
Z_{k}= \begin{cases}\alpha_{k}^{(n)} / \alpha_{\max }^{(n)} & \text { with probability } p_{k} \\ 0 & \text { otherwise }\end{cases}
$$

for each $k=1, \ldots, n$. For $k>n$ set $Z_{k} \equiv 0$. For the constants $c_{k}$ put

$$
c_{k}= \begin{cases}\alpha_{\max }^{(n)} & \text { for } k \leq n \\ 0 & \text { otherwise }\end{cases}
$$

\footnotetext{
10 The argumentation follows Dubey and Shapley (1979). (See their Sect. 11 for a convergence analysis of the Banzhaf index.)
} 
From (43) follows

$$
s=\sqrt{n} \alpha_{\max }^{(n)}
$$

This setting allows us to identify the $\tilde{Z}$ in (44) with $\alpha^{(n)}(S)-\mu^{(n)}$ and from (45) follows

$$
\operatorname{Prob}\left\{\left|\alpha^{(n)}(S)-\mu^{(n)}\right|>\delta s\right\} \leq \exp \left[-\frac{\delta^{2}}{2}\right]
$$

for any positive number $\delta>0$. Putting $\varepsilon:=\delta s$ yields the reformulation

$$
\operatorname{Prob}\left\{\left|\alpha^{(n)}(S)-\mu^{(n)}\right|>\varepsilon\right\} \leq \exp \left[-\left(\frac{\varepsilon}{2 \sqrt{n} \alpha_{\max }^{(n)}}\right)^{2}\right]
$$

Finally, for each $q \notin P$ and sufficiently large $n$ (such that $\mu^{(n)}$ is sufficiently close to $\mu$ ) each term Prob $\left\{\alpha^{n}(S) \geq q-w_{B}\right\}$ in (18) has an upper bound as given by the right-hand side of (48).

\section{References}

Baldwin R, Berglöf E, Giavazzi F, Widgren M (2000) EU reforms for tomorrow's Europe. Centre for Economic Policy Research. Discussion Paper No. 2623, London

Banzhaf JF (1965) Weighted voting doesn't work: a mathematical analysis. Rutgers Law Rev 19:317-343

Coleman JS (1971) Control of collectivities and the power of a collectivity to act. In: Lieberman B (ed) Social Choice. Gordon and Breach, New York. Reprinted in Coleman JS (1986), Individual interests and collective action, Cambridge University Press, Cambridge

Dubey P, Shapley LS (1979) Mathematical properties of the Banzhaf power index. Math Operat Res 4(2):99-131

Felsenthal DS, Machover M (1998) The measurement of voting power: theory and practise. Problems and paradoxes, Edward Elgar, Cheltenham

Felsenthal DS, Machover M (2000) Enlargement of the EU and weighted voting in its council of ministers [online], LSE research online, London. Available at: http://eprints.1se.ac.uk/archive/00000407

Felsenthal DS, Machover M (2001) The Treaty of Nice and qualified majority voting. Social Choice Welfare 18:431-464

Galloway D (2001) The Treaty of Nice and beyond: realities and illusions of power in the EU. Sheffield Academic Press, Sheffield

Hoeffding W (1963) Probability inequalities for sums of bounded random variables. J Am Stat Assoc 58:13-30

Kemperman JHB (1964) Probability methods in the theory of distribution modulo one. Compositio Math 16:106-137

Laruelle A, Valenciano F (2006) Bargaining in committees as an extension of Nash's bargaining theory. Social Choice Welfare, in press

Leech D (2002a) Designing the voting system for the council of the European Union. Public Choice 113:437-464

Leech D (2002b) Voting power in the governance of the International Monetary Fund. Ann Oper Res 109:375-397

Mann I, Shapley LS (1960) Values of large games, IV: evaluating the Electoral College by Monte Carlo techniques, The RAND Corporation, Memorandum RM-2641 (ASTIA No. AD 246277), September 19 
Petrov VV (1975) Sums of independent random variables. Springer, Heidelberg

Rae DW (1969) Decision rules and individual values in constitutional choice. Am Polit Sci Rev 63:40-56

Roth AE (1988) Introduction to the Shapley value. In: Roth AE (ed), The Shapley value. Cambridge University Press, Cambridge

Shapiro NZ, Shapley LS (1978) Values of large games, I: a limit theorem. Math Oper Res 3(1):1-9

Shapley LS (1953) A value for $n$-person games. In: Kuhn HW, Tucker AW (eds, 1953), Contributions to the theory of games II, Annals of Mathematics Studies 28. Princeton University Press, Princeton Reprinted in Roth AE (ed. 1988), The Shapley value. Cambridge University Press, Cambridge

Taylor AD, Zwicker WS (1992) A characterization of weighted voting. Proc Am Math Soc 115:1089-1094

Taylor AD, Zwicker WS (1999) Simple games: desirability relations, trading, and pseudoweightings. Princeton University Press, New Jersey

Treaty of Nice (2001) Conference of the Representatives of the Governments of the Member States, Brussels, 28 February 2001. Treaty of Nice amending the Treaty on European Union, the Treaties establishing the European Communities and certain related Acts. EU document CONFER 4820/00 